\title{
Generalization of Feedback Error Learning (FEL) to MIMO Systems
}

\author{
Alali BaSel ${ }^{*}$, Kentaro Hirata* and Kenji Sugimoto*
}

\begin{abstract}
This paper studies MIMO (Multi Input Multi Output) learning control systems. It generalizes the well-known Feedback Error Learning (FEL) scheme to MIMO systems which are not necessarily biproper, and hence not invertible with properness. Assuming that a diagonal interactor is known, a pre-filter and an exact feedforward controller are designed to achieve FEL. Furthermore, adaptive feedforward controller based on FEL is derived using linear parameterization. Stability of the adaptive control law is proved with and without positive realness condition for strictly proper and biproper systems, respectively. Simulation results illustrate the effectiveness of the proposed method.
\end{abstract}

Key Words: learning control, feedback error learning, adaptive control, MIMO systems, stability analysis

\section{Introduction}

The origin of learning feedforward control can be traced back to a scheme known as Feedback Error Learning (FEL) proposed by Kawato et al. ${ }^{1)}$. The main objective of FEL is to implement the feedforward controller as a function approximator improving approximation on-line, instead of designing linear control on the basis of a plant model. The types of function approximators used in references 1) and 15) as an example are Multi Layer Perception (MLP) and Cerebellar Model Articulation Controller (CMAC) neural networks, respectively.

Several problems exist for this kind of function approximator such as slow convergence, local minima, computational load and high memory requirement ${ }^{8)}$. However, applications have shown that the FEL controller gives a considerable improvement on the performance of the system $\left.\left.\left.\left.{ }^{1)}, 5\right), 9\right), 11\right), 15\right)$. The results in literature have shown that FEL has capabilities to improve the tracking of the desired trajectory significantly without extensive modeling. The novelty of FEL lies in its use of feedback error as a learning signal, which is essentially new in control literature ${ }^{2), 3)}$.

Recently, Miyamura and Kimura ${ }^{2)}$ have established a control theoretical validity of the FEL method in the frame of adaptive control, proving its stability based on strictly positive realness, whereas Muramatsu and Watanabe $^{3)}$ have relaxed the positive realness condition of FEL by using the error signal between the reference and the

\footnotetext{
* Nara Institute of Science and Technology, 8916-5 Takayama-cho, Ikoma City, Nara (Received July 18, 2006)

(Revised December 28, 2006)
}

output signal as well as the feedback input. Unlike 1), references 2),3) have used an adaptive linear filter for function approximator, and have not used MLP nor CMAC neural networks any more, but still the learning of the inverse model has been effectively used in references 2), 3).

The objectives of the present paper are two-folds. First, in view of the fact that most of the process control applications are multi-input multi-output (MIMO), we generalize FEL for a MIMO system in this paper. Stability of the derived MIMO-FEL learning law for square case is proved by using the idea of linear parameterization which was given by references 2),3) for SISO systems. The parameter has a matrix form instead of a vector form, but we can derive a learning law in a similar way as in the SISO case.

Secondly, we also relax the biproper condition even in the MIMO case. The concept of the interactor (Wolovich and Falb ${ }^{13)}$ ) is used for our purpose. This concept was originally introduced to generalize the relative degree of scalar transfer functions to MIMO systems.

The remainder of the paper is organized as follows. In Section 2, we formulate the problem for a MIMO system utilizing the interactor concept. In Section 3, generalization of the FEL for a MIMO system is presented. The proposed method of finding the inverse of transfer matrix is introduced exactly and adaptively. In Section 4, a learning law is derived for a MIMO system and also its stability has been studied. In Section 5 , simulation results are shown to evaluate the effectiveness of the proposed method and summarize the procedure. Section 6 summarizes the main achievements of this paper.

\section{Problem Formulation}

Consider an $n$-dimensional controllable and observable 
linear system

$$
\dot{x}(t)=A x(t)+B u(t), y(t)=C x(t)+D u(t)
$$

with $m$-inputs and $m$-outputs (i.e., square system). Assume that the system is minimum phase. Namely, we assume

$$
\operatorname{det}\left[\begin{array}{cc}
A-s I & B \\
C & D
\end{array}\right] \neq 0, \text { for all } \operatorname{Re}(s) \geq 0 .
$$

If, further, det $D \neq 0$, then the system is invertible with properness and hence generalization of FEL is straightforward, so that our method below is significantly simplified. On the other hand, if det $D=0$, then the generalization is rather difficult, since we need to define an appropriate pre-filter. We will overcome the difficulty below. In order to explain our method clearly, we will further assume $D=0$ in what follows. (If $\operatorname{det} D=0$ and $D \neq 0$, then the argument will be more complicated but our basic idea still holds.)

Therefore, the transfer matrix is $P(s)=C(s I-A)^{-1} B$. Also, we assume that $A, B, C$ are unknown, but that

$$
\Lambda:=\left[\begin{array}{c}
c_{1} A^{\mu_{1}-1} B \\
\vdots \\
c_{m} A^{\mu_{m}-1} B
\end{array}\right]
$$

is invertible, where $c_{k}$ is the $k^{t h}$ row vector of $C$ and $\mu_{k}$ is the minimal integer such that

$$
c_{k} A^{\mu_{k}-1} B \neq 0, \mu_{k} \geq 1 .
$$

The integers $\mu_{1}, \mu_{2}, \cdots, \mu_{m}$ can be regarded as a generalization of the relative degree. We assume that these integers are known. Furthermore, they have a close relationship to the following concept (Wolovich and Falb ${ }^{13)}$ ).

Definition 1. Given $P(s)$, a square polynomial matrix $L(s)$ is called an interactor if

$$
\lim _{s \rightarrow \infty} L(s) P(s)
$$

is a finite matrix with full rank.

Under the assumption that $\Lambda$ in (3) is invertible, we can set the interactor matrix to a diagonal form

$$
L(s)=\left[\begin{array}{ccc}
\left(s+a_{1}\right)^{\mu_{1}} & & 0 \\
& \ddots & \\
0 & & \left(s+a_{m}\right)^{\mu_{m}}
\end{array}\right]
$$

where $\mu_{k}$ is as defined before, and $a_{k}$ is an arbitrarily chosen positive real number for $k=1, \cdots, m$.

The feedback error learning architecture of the MIMO system is shown in Fig. 1. The objective of the control is

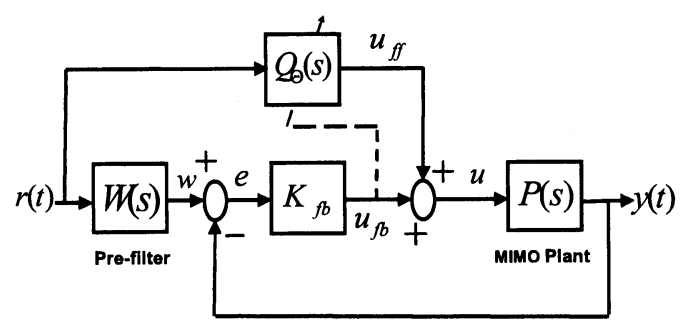

Fig. 1 MIMO Feedback Error Learning Architecture

to minimize the error signal between $w(t)$ and the plant output $y(t)$. The following equations can be deduced from Fig.1:

$$
\begin{aligned}
& y(t)=P(s) u(t) \\
& u(t)=u_{f b}(t)+u_{f f}(t) \\
& u_{f b}(t)=K_{f b} e(t), e(t)=w(t)-y(t) \\
& w(t)=W(s) r(t) \\
& u_{f f}(t)=Q_{\Theta}(s) r(t),
\end{aligned}
$$

where $K_{f b}$ is a feedback gain to stabilize the plant, and $\Theta$ is a tunable parameter. The first equation implies

$$
y(t)=£^{-1}[P(s)](t) * u(t),
$$

where $*$ denotes the time-domain convolution. For the sake of simplicity, we will adopt this kind of abuse in time and frequency domains throughout this paper. We first note that, if the system is invertible with properness as in $2), 3)$, then we can readily take $Q_{\Theta}=P^{-1}$ and obtain a perfect tracking. This is not the case, however, since we have assumed $P(s)$ is strictly proper, and hence $P^{-1}(s)$ becomes improper. In the next section, we will see how this difficulty is overcome by means of a pre-filter $W(s)$ and the concept of the interactor.

\section{Proposed Method}

We now introduce exact and adaptive methods for finding the inverse of the system. First, we set up $Q(s)$ for the exact case using the interactor concept ${ }^{13)}$. Second, we establish an adaptive method to adjust the parameter $\Theta$ in $Q_{\Theta}(s)$ of equation (7) when $A, B, C$ of the plant are assumed to be unknown. After that, we combine both methods to derive a tuning rule using the gradient descent concept.

\section{1 Exact Feedforward Controller}

For the original system (1) and the interactor $L(s)$ in (6) with arbitrarily chosen $a_{k}(k=1, \cdots, m)$, it is well known that there exists a gain $R$ such that

$$
N(s)=C(s I-A+B R)^{-1} B=L(s)^{-1} \Lambda .
$$

The gain $R$ can be obtained by the simple formula ( $\mathrm{Mu}-$ toh $\left.^{12)}\right)$ : 


$$
R:=\Lambda^{-1}\left[L_{0}, \cdots, L_{\mu}\right]\left[\begin{array}{c}
C \\
C A \\
\vdots \\
C A^{\mu}
\end{array}\right]
$$

where

$$
\begin{aligned}
& L(s)=L_{0}+L_{1} s+\cdots+L_{\mu} s^{\mu}, \\
& \mu:=\max \left(\mu_{1}, \cdots, \mu_{m}\right) .
\end{aligned}
$$

Take

$$
\begin{aligned}
W(s) & =N(s) \Lambda^{-1}=L(s)^{-1} \\
& =\left[\begin{array}{ccc}
\frac{1}{\left(s+a_{1}\right)^{\mu_{1}}} & & 0 \\
& \ddots & \\
0 & & \frac{1}{\left(s+a_{m}\right)^{\mu_{m}}}
\end{array}\right]
\end{aligned}
$$

Based on the above, we derive the following lemma.

Lemma 1: If the feedforward controller $Q(s)$ is defined by

$$
Q(s)=\left[I-R(s I-A+B R)^{-1} B\right] \Lambda^{-1} .
$$

then

$$
P(s) Q(s)=W(s) .
$$

Proof: Given in Appendix 1.

Lemma 1 means that the cascade connection (14) makes the response as simple as possible. $W(s)$ in (12) represents inevitable delay due to strictly properness of the plant. We thus take as the exact feedforward control:

$$
u_{0}(t)=Q(s) r(t) .
$$

Since $A, B, C$ of the system are assumed to be unknown, we need to adjust $Q(s)$ as presented in the next section.

Remark: We will use $W(s)$ and $Q(s)$ in (12), (13) as a pre-filter and a feedforward controller in Fig. 1, which is a natural generalization of Section 4 of reference 2).

\section{2 Adaptive Feedforward Controller}

In this section, we generalize FEL for a MIMO system by using adaptive observer parameterization ${ }^{10)}$. First, we consider the following dynamical system to parameterize the feedforward controller.

$$
\begin{aligned}
\dot{\eta_{1}}(t) & =A_{f} \eta_{1}(t)+B_{f} r(t) \\
\dot{\eta_{2}}(t) & =A_{f} \eta_{2}(t)+B_{f} u_{0}(t) \\
u_{0}(t) & =F_{0} \eta_{1}(t)+G_{0} \eta_{2}(t)+H_{0} r(t) \\
& =\Theta_{0} \eta(t),
\end{aligned}
$$

where

$$
\Theta_{0}=\left[\begin{array}{lll}
F_{0} & G_{0} & H_{0}
\end{array}\right], \eta=\left[\begin{array}{c}
\eta_{1}(t) \\
\eta_{2}(t) \\
r(t)
\end{array}\right] .
$$

$F_{0}, G_{0}, H_{0}$ are unknown matrices to be estimated.

We will show that the parameterization of (16) can yield an arbitrary transfer matrix from $r(t)$ to $u_{0}(t)$. Take $A_{f}$ and $B_{f}$ in a controllable canonical form as shown in (18) and (19)

$$
\begin{aligned}
& A_{f}=\left[\begin{array}{cccccccc}
0 & 1 & & 0 & & & & \\
\vdots & \ddots & & & & & 0 & \\
& \cdots & 1 & & & & \\
-f_{\nu} & & -f_{1} & & & & \\
& & & \ddots & & & \\
& & & & 0 & 1 & & 0 \\
& & & & & \vdots & \ddots & \\
& & & & & \cdots & 1 \\
& & & & -f_{\nu} & & -f_{1}
\end{array}\right] \\
& B_{f}=\left[\begin{array}{ccc}
0 & & \\
\vdots & & 0 \\
1 & & \\
& \ddots & \\
& & 0 \\
0 & & \vdots \\
& & 1
\end{array}\right],
\end{aligned}
$$

where $A_{f}$ is a stable matrix and $\left(A_{f}, B_{f}\right)$ is controllable. Then we have

$$
\begin{aligned}
u_{0}= & \left(I-G_{0}\left(s I-A_{f}\right)^{-1} B_{f}\right)^{-1} \\
& \cdot\left(H_{0}+F_{0}\left(s I-A_{f}\right)^{-1} B_{f}\right) r(t) \\
=: & Q_{\Theta_{0}}(s) r(t) .
\end{aligned}
$$

Taking into account that

$$
\left(s I-A_{f}\right)^{-1} B_{f}=S(s) \frac{1}{s^{\nu}+f_{1} s^{\nu-1}+\cdots+f_{\nu}}
$$

where

$$
S(s)=\left[\begin{array}{ccc}
1 & & \\
s & & 0 \\
\vdots & & \\
& & \\
s^{\nu-1} & & \\
& \ddots & \\
& & s \\
0 & & \vdots \\
& & s^{\nu-1}
\end{array}\right],
$$


we thus obtain

$$
u_{0}(t)=[X(s)]^{-1} Y(s) r(t),
$$

where

$$
\begin{aligned}
& X(s)=\left[\left(s^{\nu}+f_{1} s^{\nu-1}+\cdots+f_{\nu}\right) I-G_{0} S(s)\right], \\
& Y(s)=\left[\left(s^{\nu}+f_{1} s^{\nu-1}+\cdots+f_{\nu}\right) H_{0}+F_{0} S(s)\right] .
\end{aligned}
$$

It can be shown that equation (23) gives a complete representation of any transfer matrix of this dimension. Namely, by taking $F_{0}, G_{0}, H_{0}$ appropriately, we can obtain $Q(s)$ derived in the previous section, in the form of (23). In reality, however, we do not know such parameters $F_{0}, G_{0}$, and $H_{0}$, so that we regard these matrices as tunable coefficients and adjust them accordingly to some learning law.

We now construct a tunable feedforward controller. In order to generate $u_{f f}(t)$, we consider the following dynamical system, instead of (16):

$$
\begin{aligned}
\dot{\xi}_{1}(t) & =A_{f} \xi_{1}(t)+B_{f} r(t) \\
\dot{\xi}_{2}(t) & =A_{f} \xi_{2}(t)+B_{f} u_{f f}(t) \\
u_{f f}(t) & =F(t) \xi_{1}(t)+G(t) \xi_{2}(t)+H(t) r(t) \\
& =\Theta(t) \xi(t),
\end{aligned}
$$

where

$$
\Theta(t)=\left[\begin{array}{lll}
F(t) & G(t) & H(t)
\end{array}\right], \quad \xi(t)=\left[\begin{array}{c}
\xi_{1}(t) \\
\xi_{2}(t) \\
r(t)
\end{array}\right] .
$$

Note that the unknown parametric matrices $F(t), G(t)$, $H(t)$ enter linearly. The matrix $\Theta(t)$ is tuned using the learning law, which will be derived in the next section.

\section{3 Learning Law}

The learning law is derived using the gradient method with an error function, defined for each time as follows:

$$
V=\frac{1}{2}\left(u_{f f}-u_{0}\right)^{T}\left(u_{f f}-u_{0}\right)
$$

Then the steepest gradient method is formulated as

$$
\frac{d \Theta}{d t}=-\alpha \frac{\partial V}{\partial \Theta},
$$

for small $\alpha$.

A main difference from references 1) and 2) is, however, that $\Theta$ is not a vector, but a matrix. Still the idea carries over only with a slight modification.

We define

$$
\frac{\partial V}{\partial \Theta}=\left(\frac{\partial V}{\partial \theta_{i j}}\right)
$$

where

$$
\Theta=\left(\theta_{i j}\right)
$$

Thus, we calculate

$$
\frac{\partial V}{\partial \theta_{i j}}=\frac{\partial}{\partial \theta_{i j}}\left[\frac{1}{2} \sum_{k} v_{k}^{2}\right],
$$

where

$$
v=u_{f f}-u_{0}=\left(v_{1}, \cdots, v_{m}\right)^{T} .
$$

Using (24), we have

$$
v=\Theta \xi-u_{0} .
$$

Therefore

$$
\begin{aligned}
\frac{\partial V}{\partial \theta_{i j}} & =\sum_{k}\left[v_{k} \frac{\partial v_{k}}{\partial \theta_{i j}}\right] \\
& =\sum_{k}\left[v_{k} \frac{\partial}{\partial \theta_{i j}}\left[\sum_{l} \theta_{k l} \xi_{l}-u_{0 k}\right]\right] .
\end{aligned}
$$

Also, we have

$$
\frac{\partial}{\partial \theta_{i j}}\left[\sum_{l} \theta_{k l} \xi_{l}-u_{0 k}\right]=\delta_{i k} \xi_{j} .
$$

where

$$
\delta_{i k}=\left\{\begin{array}{cc}
0 & i \neq k \\
1 & i=k
\end{array} .\right.
$$

Hence, we have

$$
\frac{\partial V}{\partial \theta_{i j}}=v_{i} \xi_{j}, \quad i, j=1, \cdots, m .
$$

Therefore, we obtain

$$
\frac{\partial V}{\partial \Theta}=v \xi^{T} .
$$

We have derived (15) since $e(t)=0$, which implies that we can take $u(t)=u_{0}(t)$. The tuning rule then becomes

$$
\frac{d \Theta}{d t}=-\alpha\left(u_{f f}(t)-u(t)\right) \xi^{T}(t)=\alpha u_{f b}(t) \xi^{T}(t)
$$

where $\alpha$ is a scalar parameter introduced to adjust adaptation speed. The above learning law clearly depends on the feedback error signal and this is why we call it the feedback error learning.

\section{Stability of the Proposed Scheme}

The stability of the above differential equation (38) can be verified as in reference 2 ) using the strictly positive realness condition. Using (14), (15) and (7), we obtain the following relation

$$
\begin{aligned}
& P(s)\left[u_{0}(t)-u(t)\right] \\
& =P(s) Q(s) r(t)-P(s) u(t) \\
& =W(s) r(t)-y(t)=e(t) .
\end{aligned}
$$


We assume that the initial conditions of equations (16) and (24) are the same, then the subtraction between the first two equations of (16) and (24) yields the following relations

$$
\begin{aligned}
& \eta_{1}=\xi_{1}, \\
& \eta_{2}=\xi_{2}+\left(s I-A_{f}\right)^{-1} B_{f}\left(u_{0}(t)-u(t)\right) .
\end{aligned}
$$

Using (16), (24) and (7), we obtain the following relation

$$
u_{0}(t)-u(t)=\Theta_{0} \eta(t)-\Theta(t) \xi(t)-K_{f b} e(t) .
$$

Substituting (40) in (41), we have

$$
\begin{aligned}
\begin{aligned}
& u_{0}(t)-u(t)= \Theta_{0} \xi(t)-\Theta(t) \xi(t)-K_{f b} e(t) \\
&+G_{0}\left(s I-A_{f}\right)^{-1} B_{f}\left(u_{0}(t)-u(t)\right) \\
&=-\left[I-G_{0}\left(s I-A_{f}\right)^{-1} B_{f}\right]^{-1}\left[\Psi(t) \xi(t)+K_{f b} e(t)\right],
\end{aligned}
\end{aligned}
$$

where

$$
\Psi(t)=\Theta(t)-\Theta_{0}
$$

$\Theta_{0}$ can be regarded as the nominal value of $\Theta(t)$.

Pre-multiplying (42) by $P(s)$, we have

$$
\begin{aligned}
& P(s)\left[u_{0}(t)-u(t)\right]= \\
& -P(s)[J(s)]^{-1}\left[\Psi(t) \xi(t)+K_{f b} e(t)\right],
\end{aligned}
$$

where

$$
J(s)=\left[I-G_{0}\left(s I-A_{f}\right)^{-1} B_{f}\right]
$$

From (15) and (20), we have

$$
\begin{gathered}
{\left[I-G_{0}\left(s I-A_{f}\right)^{-1} B_{f}\right]^{-1}=} \\
Q(s) \cdot\left[H_{0}+F_{0}\left(s I-A_{f}\right)^{-1} B_{f}\right]^{-1} .
\end{gathered}
$$

Substituting (39) and (45) in (44), we have

$$
\begin{aligned}
& e(t)=-P(s) Q(s)\left[H_{0}+\right.\left.F_{0}\left(s I-A_{f}\right)^{-1} B_{f}\right]^{-1} \\
& \cdot\left[\Psi(t) \xi(t)+K_{f b} e(t)\right] .
\end{aligned}
$$

By (14), we obtain

$$
\begin{aligned}
e(t)= & -\left[I+W(s)\left(H_{0}+F_{0}\left(s I-A_{f}\right)^{-1} B_{f}\right)^{-1} K_{f b}\right]^{-1} \\
& \cdot W(s)\left[H_{0}+F_{0}\left(s I-A_{f}\right)^{-1} B_{f}\right]^{-1} \Psi(t) \xi(t) \\
= & -\left[\left(H_{0}+F_{0}\left(s I-A_{f}\right)^{-1} B_{f}\right) L(s)+K_{f b}\right]^{-1} \\
& \cdot \Psi(t) \xi(t)
\end{aligned}
$$

Substituting $e(t)$ in the derived learning law (38), we obtain

$$
\begin{gathered}
\frac{d \Theta}{d t}=-\alpha K_{f b}\left[\left(H_{0}+F_{0}\left(s I-A_{f}\right)^{-1} B_{f}\right) L(s)\right. \\
\left.+K_{f b}\right]^{-1} \Psi(t) \xi(t) \xi(t)^{T} .
\end{gathered}
$$

Namely, we have

$$
\frac{d \Psi}{d t}=-\alpha M(s) \Psi(t) \xi(t) \xi^{T}(t),
$$

where

$$
\begin{gathered}
M(s)=K_{f b}\left[\left(H_{0}+F_{0}\left(s I-A_{f}\right)^{-1} B_{f}\right) L(s)+K_{f b}\right]^{-1} \\
=K_{f b} W(s)\left[H_{0}+F_{0}\left(s I-A_{f}\right)^{-1} B_{f}+K_{f b} W(s)\right]^{-1}
\end{gathered}
$$

Following the idea in reference 2), we show the following lemma.

Lemma 2: Let $M(s)$ be strictly positive real transfer matrix and $\xi(t)$ be an arbitrary time-varying vector. Then, the solution $\Psi(t)$ of (49) tends to a constant matrix $\Psi_{0}$ such that $\Psi_{0} \xi(t) \rightarrow 0$. If $\xi(t)$ satisfies the so-called persistent excitation (PE) condition, the above $\Psi_{0}$ is equal to zero matrix.

Proof: Consider the state space representation of (49)

$$
\begin{aligned}
\frac{d x(t)}{d t} & =\hat{A} x(t)+\hat{B} \Psi(t) \xi(t) \\
y(t) & =\hat{C} x(t)+\hat{D} \Psi(t) \xi(t) \\
\frac{d \Psi(t)}{d t} & =-y(t) \xi^{T}(t) .
\end{aligned}
$$

Since $M(s)$ is strictly positive real, it implies that there exist $P=P^{T}>0, T$, and $Z$, and a positive constant $\epsilon$ given in KYP lemma (see Appendix). We consider the following Lyapunov function

$$
V(t)=\operatorname{tr}\left\{\Psi^{T}(t) \Psi(t)\right\}+x^{T}(t) P x(t) .
$$

Taking the derivative

$$
\begin{aligned}
\dot{V}(t)= & \operatorname{tr}\left\{\Psi^{T}(t) \dot{\Psi}(t)\right\}+\operatorname{tr}\left\{\dot{\Psi}^{T}(t) \Psi(t)\right\} \\
& +\dot{x}^{T}(t) P x(t)+x^{T}(t) P \dot{x}(t) .
\end{aligned}
$$

Using KYP lemma, we have (for $x \neq 0$ )

$$
\dot{V}(t)=-\|T x(t)+Z \Psi(t) \xi(t)\|^{2}-\epsilon x^{T}(t) P x(t)<0 .
$$

Since $V(t) \geq 0$ while $\dot{V}(t)<0$, this implies that $x(t)$ and $\Psi(t) \xi(t)$ converge to 0 . Also, as $x(t) \rightarrow 0$, by (51) we have

$$
\frac{d \Psi(t)}{d t}=-\hat{D} \Psi(t) \xi(t) \xi^{T}(t)
$$

As a result, if $\xi(t)$ satisfies PE then the above differential equation is globally exponentially stable $\Psi(t) \rightarrow 0$ based on the PE and Exponential Stability Theorem in reference 14$)$. $\diamond$

As a result, we state the following theorem:

Theorem 1: Note that

$$
\begin{aligned}
Q_{\Theta}(s)= & \left(I-G(t)\left(s I-A_{f}\right)^{-1} B_{f}\right)^{-1} \\
& \cdot\left(H(t)+F(t)\left(s I-A_{f}\right)^{-1} B_{f}\right) .
\end{aligned}
$$


Under any stable transfer matrix $P(s)$ with minimum phase and known upper bound of the relative degree $\left(\mu:=\max \left(\mu_{1}, \cdots, \mu_{m}\right)\right)$, the MIMO-FEL scheme and its learning law are stable if $K_{f b}$ is chosen such that $M(s)$ in (49) is strictly positive real. Also, it yields $e(t) \rightarrow 0$, and $Q_{\Theta}(s) r(t) \rightarrow Q(s) r(t)$ if $\xi(t)$ satisfies the PE condition.

Proof: Based on lemma 2, if $K_{f b}$ is chosen such that $M(s)$ is strictly positive real, then the solution of the adaptive law based on FEL will tend to a constant matrix which proves the stability. Also, if $\xi(t)$ satisfies the PE condition, then $\Psi(t) \rightarrow 0$ and $e(t) \rightarrow 0$ as time proceeds and ends up with $u_{f f} \rightarrow u_{0}$.

The above result is a generalization of reference 2). A possible drawback in this approach is that it requires positive realness condition, which is not always guaranteed.

Following reference 3), we establish the stability of the FEL without the positive realness condition in the case of biproper systems, (i.e. $W(s)=I$ ). The main idea is how to make $M(s)$ being equal to the identity matrix by influencing sort of dynamics which is based on the error signal. The new vector $\xi_{e}(t)$ is generated by $e(t)$ with the following dynamics

$$
\dot{\xi}_{e}(t)=A_{f} \xi_{e}(t)+B_{f} e(t)
$$

where $A_{f}$ and $B_{f}$ are as defined before.

From (46) and taking Laplace transform of (57), we have the following relation

$$
\begin{aligned}
& {\left[H_{0}+F_{0}\left(s I-A_{f}\right)^{-1} B_{f}-K_{f b}\right] e(t)} \\
& =\left[H_{0}-K_{f b}\right] e(t)+F_{0} \xi_{e}(t) .
\end{aligned}
$$

Then, substitute (58) into (45), and define

$$
\zeta:=\left[\begin{array}{c}
\xi_{1}(t)+\xi_{e}(t) \\
\xi_{2}(t) \\
r(t)
\end{array}\right]
$$

we obtain

$$
\hat{u}_{0}(t)=\Theta_{0} \zeta(t)
$$

which is linearly parameterized by $\Theta_{0}$. In order to derive an adaptive law for the FEL, we define $\hat{u}(t)$ by replacing $\Theta_{0}$ in $(60)$ with $\Theta(t)$

$$
\hat{u}(t)=\Theta(t) \zeta(t) .
$$

We now define an error signal $s(t)$ as

$$
s(t):=\hat{u_{0}}(t)-\hat{u}(t)=-\Psi(t) \zeta(t) .
$$

Using the gradient method as we did before with error function $s(t)$, the learning law turns out to be

$$
\frac{d \Theta(t)}{d t}=\alpha s(t) \zeta^{T}(t) .
$$

Using (43) and (62), the learning law can be written as

$$
\frac{d \Psi(t)}{d t}=-\alpha \Psi(t) \zeta(t) \zeta^{T}(t) .
$$

The stability of the above differential equation (64) can be verified using the following lemma.

Lemma 3: Let $\zeta(t)$ be an arbitrary time-varying vector. Then, the solution of the differential equation (64) tends to a constant matrix $\Psi_{0}$ such that $\Psi_{0} \zeta(t) \rightarrow 0$. If $\zeta(t)$ satisfies the persistent excitation (PE) condition, then the above matrix $\Psi_{0}$ is equal to zero matrix.

Proof: Let the Lyapunov function defined as follows

$$
V(t)=\frac{1}{2} \operatorname{tr}\left[\Psi^{T}(t) \Psi(t)\right] .
$$

Then, the derivative along the trajectory is given by

$$
\begin{aligned}
\dot{V}(t) & =\operatorname{tr}\left[\Psi^{T}(t) \dot{\Psi}(t)\right] \\
& =-\operatorname{tr}\left[\Psi^{T}(t) \Psi(t) \zeta(t) \zeta^{T}(t)\right] \leq 0 .
\end{aligned}
$$

Since $V(t) \geq 0$ while $\dot{V}(t) \leq 0$, this implies that the above differential equation is stable according to Lyapunov theorem. Therefore, $\Psi(t) \zeta(t)$ converges to zero vector and thus $\frac{d \Psi(t)}{d t} \rightarrow 0$. For the second part of lemma 2 , if $\zeta(t)$ is PE then the learning law is globally exponentially stable, namely $\Psi(t) \rightarrow 0$ based on the PE and Exponential Stability Theorem ${ }^{14)}$.

Thus we derive the following theorem:

Theorem 2: Under any stable transfer matrix $P(s)$ with minimum phase and known upper bound of the relative degree $\left(\mu:=\max \left(\mu_{1}, \cdots, \mu_{m}\right)\right)$, the MIMO-FEL scheme and its learning law (64) are stable. Also, it yields $e(t) \rightarrow 0$ if $\zeta(t)$ satisfies the $\mathrm{PE}$ condition.

Proof: From lemma $3 \Psi(t)$ tends to constant matrix $\Psi_{0}$ such that $\Psi_{0} \zeta(t) \rightarrow 0$. Therefore, $s(t)=-\Psi(t) \zeta(t) \rightarrow 0$. If $\zeta(t)$ satisfies the PE condition then $\Psi_{0}$ is equal to $\mathbf{0}$, and then $\Theta(t) \rightarrow \Theta_{0}$ using (43). This implies that $e(t) \rightarrow 0$ as time proceeds. $\diamond$

Corollary: When $e(t) \rightarrow 0$ as $t \rightarrow \infty$, then

$$
\Theta(t) \rightarrow \Theta_{0}, \text { and } Q_{\Theta}(s) r(t) \rightarrow Q(s) r(t) .
$$

The corollary concludes that the adaptive feedforward control based on MIMO-FEL will match the exact one which is based on interactorization concept as time proceeds.

Remark: $A$ vector $\zeta(t)$ is persistently exciting (PE) if there exist $\lambda_{1}, \lambda_{2}, \delta>0$ such that 


$$
\lambda_{1} I \geq \int_{t_{0}}^{t_{0}+\delta} \zeta(t) \zeta^{T}(t) d t \geq \lambda_{2} I, \text { for all } t_{0} \geq 0
$$

as defined in reference 10).

\section{Simulation Results}

In simulation below, we sometimes use $u(t)$ instead of $u_{f f}(t)$ in (24) with slight abuse ${ }^{2), 3)}$. First we assume that $A, B, C$ are known so that we can calculate the exact feedforward controller $Q(s)$. Then, we will use the learning law to adjust tunable $\Theta(t)$ and compare it with nominal one.

Giving

$$
\begin{aligned}
& A=\left[\begin{array}{rrr}
1 & 0 & -3 \\
2 & -4 & 1 \\
3 & 1 & -5
\end{array}\right], B=\left[\begin{array}{ll}
0 & 0 \\
1 & 0 \\
0 & 1
\end{array}\right], \\
& C=\left[\begin{array}{lll}
1 & 1 & 0 \\
0 & 2 & 1
\end{array}\right]
\end{aligned}
$$

we have the following transfer matrix of the plant:

$$
P(s)=\left[\begin{array}{cc}
\frac{s^{2}+4 s+1}{s^{3}+8 s^{2}+19 s+23} & \frac{-2 s-19}{s^{3}+8 s^{2}+19 s+23} \\
\frac{2 s^{2}+9 s+7}{s^{3}+8 s^{2}+19 s+23} & \frac{s^{2}+5 s-18}{s^{3}+8 s^{2}+19 s+23}
\end{array}\right] .
$$

Using (3), we have $\mu_{1}=1, \mu_{2}=1$, and

$$
\Lambda=\left[\begin{array}{ll}
1 & 0 \\
2 & 1
\end{array}\right]
$$

which is invertible. Then, we select the interactor

$$
L(s)=\left[\begin{array}{cc}
s+2 & 0 \\
0 & s+2.5
\end{array}\right] .
$$

We now calculate the gain $\mathrm{R}$ in order to find the exact $Q(s)$ using equations (9) and (13):

$$
\begin{aligned}
& R=\left[\begin{array}{rrr}
5 & -2 & -2 \\
-3 & 2 & 3
\end{array}\right] \\
& Q(s)=\left[\begin{array}{cc}
\frac{s^{2}+5 s-18}{s^{2}+7 s+10} & \frac{2 s+19}{s^{2}+7.5 s+12.5} \\
\frac{-2 s^{2}-9 s-7}{s^{2}+7 s+10} & \frac{s^{2}+4 s+1}{s^{2}+7.5 s+12.5}
\end{array}\right] .
\end{aligned}
$$

It can be verified that $P(s) Q(s)=W(s)=L^{-1}(s)$.

Second, we tune $\Theta(t)$ using the learning rule (38) so that we obtain the inverse of the system provided that the reference inputs are sine signals $\left(r(t)=[\sin (t), \sin (t)]^{T}\right)$. Thus, we need to set stable and controllable $A_{f}$ and $B_{f}$ based on the upper bound of the relative degree (11) $\mu=1$, (see (18) and (19)),

$$
A_{f}=\left[\begin{array}{rr}
-4 & 0 \\
0 & -4
\end{array}\right], B_{f}=\left[\begin{array}{ll}
1 & 0 \\
0 & 1
\end{array}\right] \text {. }
$$

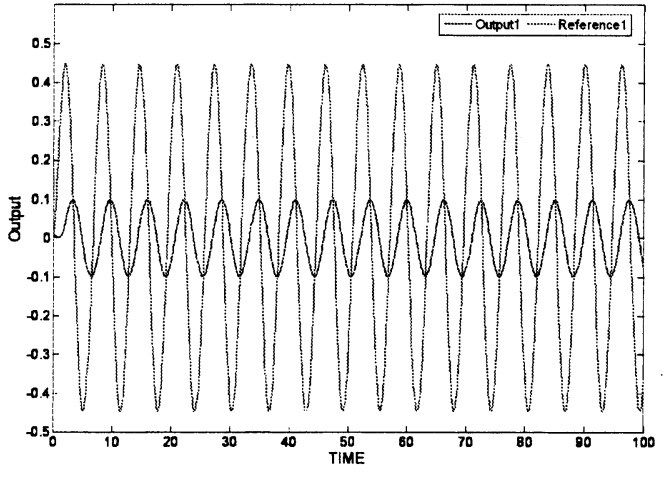

Fig. 2 Reference vs. Output without MIMO-FEL:Channel 1

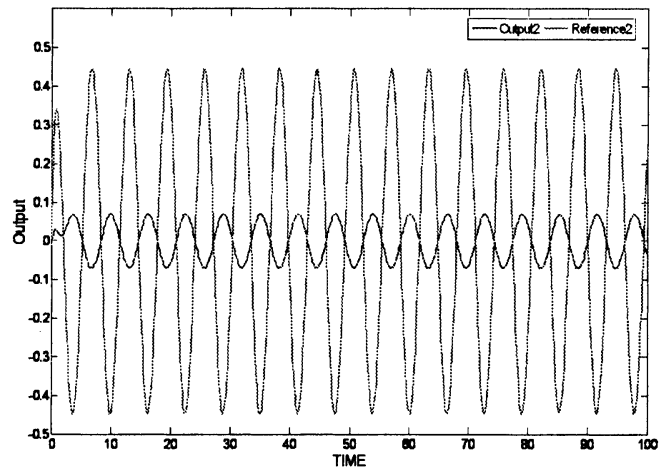

Fig. 3 Reference vs. Output without MIMO-FEL:Channel 2

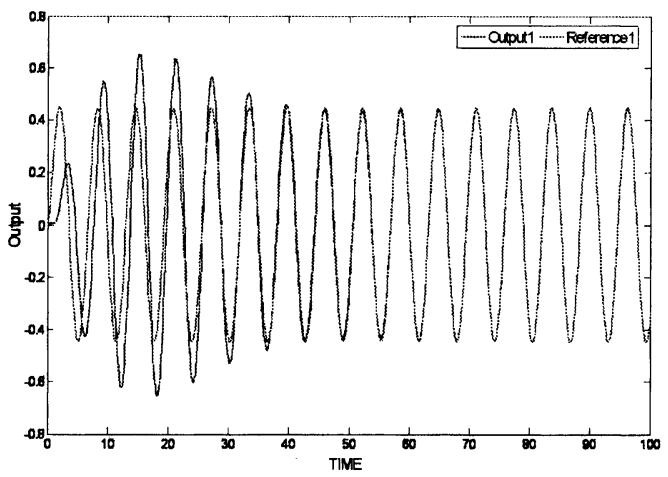

Fig. 4 Reference vs. Output with MIMO-FEL: Channel 1

We choose the following feedback gain matrix that maintains the closed-loop stability and satisfy SPR condition

$$
K_{f b}=\left[\begin{array}{rr}
0.1 & 0.25 \\
-0.3 & -0.15
\end{array}\right]
$$

The simulation results in Figs. 2 and $\mathbf{3}$ using only feedback controller show that the tracking performance was not really good. However, it is shown in Figs. 4 and $\mathbf{5}$ that all outputs $y(t)$ track their reference inputs $w(t)$ using the proposed MIMO-FEL. Also, it is shown in Fig. 6 that the error signals tend to zero for MIMO-FEL performance. Furthermore, all the parameters $F(t), G(t)$ and $H(t)$ which correspond to $\Theta(t)$ converge to their nominal 


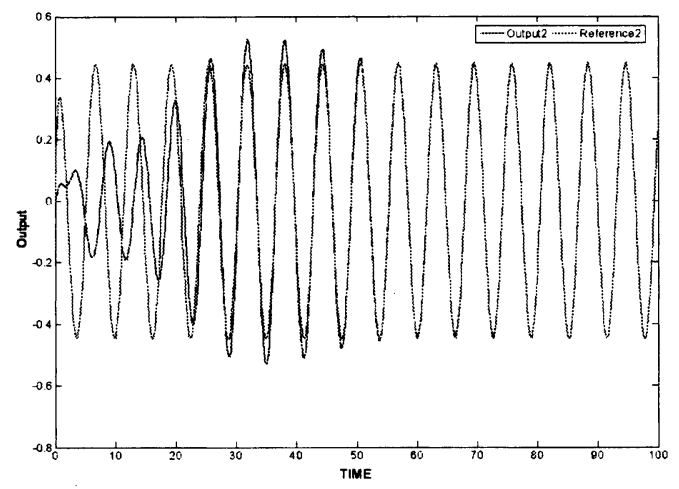

Fig. 5 Reference vs. Output with MIMO-FEL: Channel 2

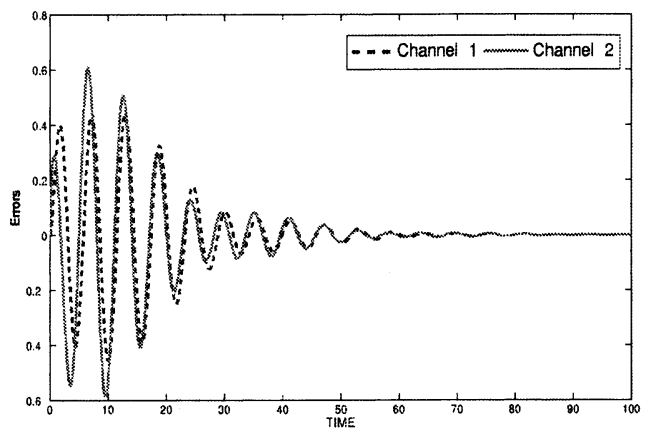

Fig. 6 Tracking Errors for MIMO-FEL

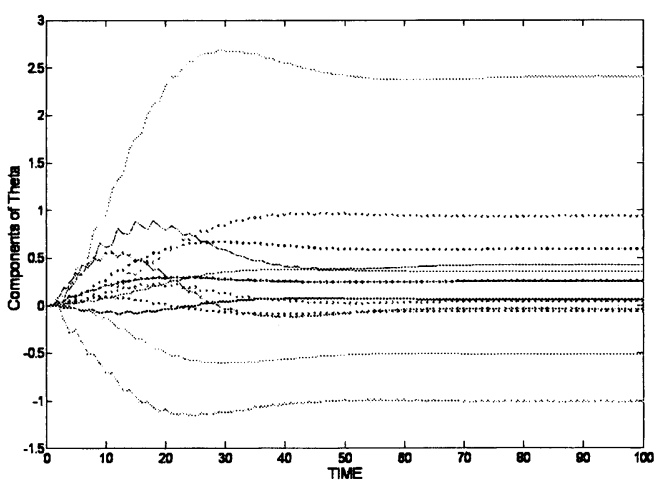

Fig. 7 Time Evolution of $\Theta(t)$

values since $e(t) \rightarrow 0$ as shown in Fig. 7. The resulting matrices are as follows

$$
\begin{aligned}
& F_{0}=\left[\begin{array}{rr}
0.5888 & 0.1636 \\
-0.0856 & 0.2545
\end{array}\right], \\
& G_{0}=\left[\begin{array}{rr}
0.6524 & -0.0452 \\
-0.1542 & 0.1728
\end{array}\right], \\
& H_{0}=\left[\begin{array}{rr}
-0.0032 & 2.5197 \\
-1.0685 & -0.0875
\end{array}\right] .
\end{aligned}
$$

By substituting the above matrices and the defined $A_{f}$ and $B_{f}$, the estimated transfer matrix can be easily obtained as follows

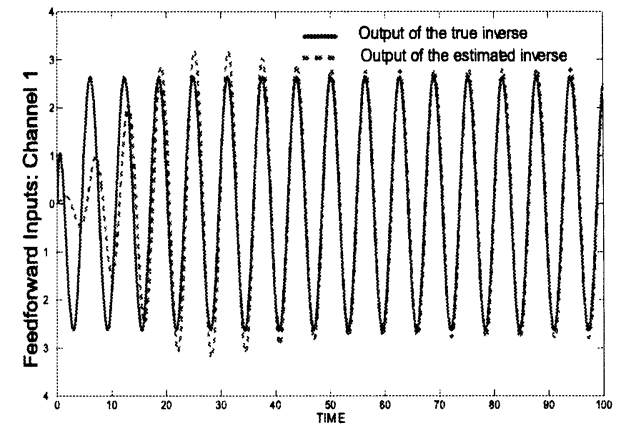

Fig. 8 Feedforward Inputs $u_{f f}, u_{f f}^{*}$ : Channel 1

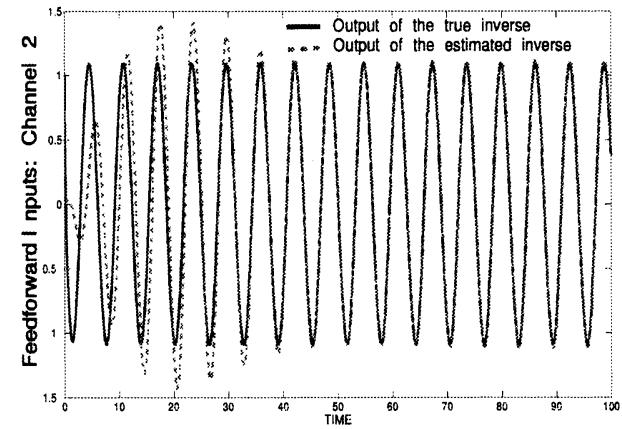

Fig. 9 Feedforward Inputs $u_{f f}, u_{f f}^{*}$ : Channel 2

$$
\begin{gathered}
Q_{\Theta}(s)=\left[I-G_{0}\left(s I-A_{f}\right)^{-1} B_{f}\right]^{-1} \\
\left.\cdot\left[H_{0}+F_{0}\left(s I-A_{f}\right)^{-1}\right) B_{f}\right] \\
=\left[\begin{array}{cc}
\frac{0.4188 s^{2}+4.314 s+10.39}{s^{2}+6.721 s+11.23} & \frac{2.39 s^{2}+18.4 s+35.12}{s^{2}+6.721 s+11.23} \\
\frac{-1.013 s^{2}-7.22 s-12.59}{s^{2}+6.721 s+11.23} & \frac{-0.0455 s^{2}+0.001115 s+0.5082}{s^{2}+6.721 s+11.23}
\end{array}\right] .
\end{gathered}
$$

It should be noted that the exact $Q(s)(69)$ is the true inverse of the plant while the $Q_{\Theta}(s)(70)$ is an estimated inverse for the specified reference inputs $r(t)$. This means that for different $r(t)$, we will obtain different estimated inverse. It seems that this is because of lack of PE in this case. In Figs. 8 and $\mathbf{9}$, we compare the outputs of the true inverse and the estimated one against $r(t)$ :

$$
\begin{aligned}
& u_{f f}^{*}=Q(s) r, \\
& u_{f f}=Q_{\Theta}(s) r .
\end{aligned}
$$

It is shown that the adaptive feedforward inputs approach to the exact one which clarify the obtained corollary. This concludes that the adaptive rule successfully learns the command for given tracking of the MIMO system, the main goal of this paper.

\section{Conclusion}

In this paper, learning control structures are proposed for a MIMO systems using Feedback Error Learning. By using linear system parameterization as a function ap- 
proximator of the feedforward control, we derive a learning law to adjust the inverse of the plant. A theoretical treatment of how to generalize feedback error learning (FEL) to MIMO systems has been studied in the framework of adaptive control. The FEL scheme is generalized to MIMO systems which are not necessarily biproper, and hence not invertible with properness. The feedforward controller is not designed on the basis of the process model. However, it is trained on-line during control using feedback error signal as a learning signal.

Moreover, the exact inverse of the plant has been derived theoretically using inverse interactorization concept, and can be used as a guide to check the correctness of the learning control. Furthermore, the stability of the tuning rule has been proved with and without positive realness condition.

The simulation results have shown that MIMO-FEL scheme has the capability to improve the system performance drastically.

\section{References}

1) M. Kawato, K. Furukawa and R. Suzuki: A hierarchical neural-network model for control and learning of voluntary movement, Biol. Cybern., 57, 169/185 (1987)

2) A. Miyamura and H. Kimura: Stability of feedback error learning scheme, Systems Control Letters, 45, 303/316 (2002)

3) E. Muramatsu and K. Watanabe: Feedback error learning control without recourse to positive realness, IEEE Trans on Automatic Control, 49-10, 1762/1767 (2004)

4) A. Datta and J. Ochua: Adaptive internal model control: design and stability analysis, Automatica, 32, 261/266 (1996)

5) M. Kawato: Feedback-Error-Learning neural network for supervised motor learning, Advanced Neural Computers, Elsevier Sci., 365/373 (1990)

6) T. de Vries, W. Velthuis and J. Amerongen: Learning feedforward control: A survey and historical note, Proc. IFAC Conf. on Mechatronic Sys., 197/202 (2000)

7) W. Velthuis, T. de Vries, P. Schaak and E. Gaal: Stability analysis of learning feedforward control, Automatica, $\mathbf{3 6}$ $1889 / 1895(2000)$

8) K. S. Narendra and L. Valavani: Stable Adaptive Systems, C Prentice-Hall International Edition, Englewood Cliffs, NJ (1989)

9) H. Gomi and M. Kawato: Neural network control for a closed-loop system using Feedback-Error-Learning, Neural Networks, 6, 933/946 (1993)

10) K. Astrom and B. Wittenmark: Adaptive Control, Addison Wesley, Reading, MA, U.S.A (1989)

11) M. Er and K. Liew: Control of adept one SCARA robot using neural networks, IEEE Trans. Industrial Engineering, 44, 762/768 (1997)

12) $Y$. Mutoh and P. Nikiforuk: Inversed interactorizing and triangularizing with an arbitrary pole assignment using a state feedback, IEEE Trans. Automatic Control, 14 , $630 / 633$ (1992)

13) W. Wolovich and P. Falb: Invariants and canonical forms under dynamic compensation, SIAM J. Control Optimiz., 14, 996/1008 (1976)

14) S. Sastry: Adaptive Control Stability, Convergence, and Robustness, Prentice Hall, NJ, U.S.A (1989)

15) S. Ananthraman and D. Grag: Training backpropagation and CMAC neural networks for control of a SCARA robot, Engineering Applications of Artificial Intelligence, 6-2, 105/115

\section{Appendix}

\section{Proof of Lemma1:}

$$
\begin{aligned}
& P(s) Q(s)-W(s) \\
& =C(s I-A)^{-1} B\left[I-R(s I-A+B R)^{-1} B\right] \Lambda^{-1} \\
& -C(s I-A+B R)^{-1} B \Lambda^{-1} \\
& =C\left\{(s I-A)^{-1} B-(s I-A)^{-1} B R(s I-A\right. \\
& \left.+B R)^{-1} B-(s I-A+B R)^{-1} B\right\} \Lambda^{-1} \\
& =C\left\{(s I-A)^{-1} B-\left[(s I-A)^{-1} B R+I\right]\right. \\
& \left.\cdot[s I-A+B R]^{-1} B\right\} \Lambda^{-1} \\
& =C\left\{(s I-A)^{-1} B-(s I-A)^{-1}[B R+s I-A]\right. \\
& \left.\cdot[s I-A+B R]^{-1} B\right\} \Lambda^{-1} \\
& =C\left\{(s I-A)^{-1} B-(s I-A)^{-1} B\right\} \Lambda^{-1}=0 .
\end{aligned}
$$

2. KYP Lemma (Kalman-Yakubovich-Popov) ${ }^{14)}$ : Let $G(s):=D+C(s I-A)^{-1} B$

be $p \times p$ transfer matrix, where $(A, B)$ is controllable and $(A, C)$ is observable. Then, $G(s)$ is strictly positive real if and only if there exist matrices $P=P^{T}>0, T$, and $Z$, and a positive constant $\epsilon$ such that

$$
\begin{aligned}
& P A+A^{T} P=-T^{T} T-\epsilon P \\
& P B=C^{T}-T^{T} Z \\
& Z^{T} Z=D+D^{T}
\end{aligned}
$$

\section{Alali BASEL}

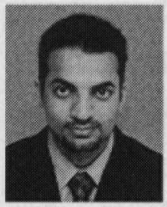

He received his BS degree in Systems Engineering from King Fahd University of Petroleum and Minerals in 2000. He was working with ACEC Engineering Center as control and Instrument Engineer in 2001-2002. In 2003, he was a research student at Nagoya University. He received his MS degree in Information Science from Nara Institute of Science and Technology (NAIST) in 2005. He is currently a $\mathrm{PhD}$ student at NAIST. His research interests include linear systems, adaptive and learning control. 


\section{Kentaro Hirata (Member)}

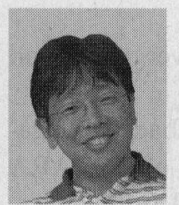

He received Master and Doctor Degrees from Kyoto University in 1990,1997 , respectively. During this period, he had a working opportunity in Sumitomo Metal Industries. After the professional career in Kyoto and Osaka Prefecture University, he joined Nara Institute of Science and Technology in 2005. He is currently Associate Professor in Graduate School of Information Science. His research interests are aimed at the way things work and are controlled. He is a member of IEEE, and ISCIE.

\section{Kenji Sugimoto (Member)}

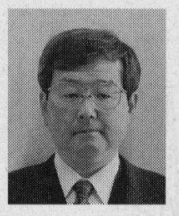

He received a Master's degree and Doctor's degree from Kyoto University in 1982, 1989, respectively. After working with Mitsubishi Electric Corporation, he became Assistant Professor at Kyoto University in 1985. Then he was Associate Professor at Okayama and Nagoya Universities. Since 1999, he has been Professor at Nara Institute of Science and Technology. His current research interests include control theory and systems science. He is a member of IEEE, ISCIE, and IEICE. 